\title{
Evidence for SAMHD1 Tumor Suppressor Functions in Acute Myeloid Leukemia
}

\author{
Torsten Schaller $^{\mathrm{a}}$ Nikolas Herold ${ }^{\mathrm{b}, \mathrm{c}}$ \\ a University Hospital, Heidelberg University, Heidelberg, Germany; ${ }^{\mathrm{b}}$ Division of Pediatric Oncology and Hematology, \\ Department of Women's and Children's Health, Karolinska Institutet, Stockholm, Sweden; 'Pediatric Oncology, \\ Theme of Children's and Women's Health, Karolinska University Hospital Solna, Stockholm, Sweden
}

\section{Editorial comment on the paper by Huinan Jiang et al. Expression and Relationship of SAMHD1 with Other Apoptotic and Autophagic Genes in Acute Myeloid Leukemia Patients. An Original Paper. Acta Haematol 2020;143:51-59}

The deoxynucleoside triphosphate triphosphohydrolase SAMHD1 is able to hydrolyze the active metabolite of the deoxycytidine analogue cytarabine, ara-CTP, and thereby decreases the sensitivity of acute myeloid leukemia (AML) cells to cytarabine (ara-C) in vitro and in vivo [1]. Clinically, SAMHD1 critically limits the efficacy of ara-C consolidation therapies in AML: 3-year overall survival in AML treated with ara-C was $>70 \%$ in patients with low SAMHD1 levels as compared to $<35 \%$ in patients with high SAMHD1 levels in their AML blasts at diagnosis $[1,2]$. Hence, SAMHD1 can be considered a bona fide resistance factor for ara-C therapy of AML.

However, contrary to its role as a resistance factor, the finding of reduced SAMHD1 expression in cutaneous Tcell lymphoma, chronic lymphocytic leukemia, as well as lung and colorectal cancer suggests that SAMHD1 harbors tumor suppressor properties (reviewed in [3]). In addition to copy number variations, several mechanisms of SAMHD1 downregulation have been described: overexpression of microRNA miR-188a and miR-155, increased SAMHD1 promoter methylation, and somatic mutations in SAMHD1 [1,3]. Mechanistically, tumor suppression exerted by SAMHD1 might be coupled to its cell cycle-dependent enzymatic activity in limiting deoxynucleoside triphosphate pools required for DNA replication. Together with other enzymes that modulate cel-

\section{KARGER}

(C) 2019 S. Karger AG, Basel

E-Mail karger@karger.com

www.karger.com/aha lular nucleotide metabolism (e.g., ribonucleotide reductase), SAMHD1 controls the balance of the intracellular dNTP pools whose dysregulation can promote tumorigenesis [3]. More recently, a report on a nonenzymatic function of SAMHD1 in the cellular response to replication stress provides another line of evidence of how SAMHD1 might impede tumorigenesis: by stimulation of the double-strand break repair protein MRE11A (MRE11) at stalled replication forks, SAMHD1 triggers the ataxia telangiectasia and Rad3-related protein (ATR)/checkpoint kinase 1 (Chk1) checkpoint and thereby prevents genomic instability [4]. Disruptions of this checkpoint in cancer cells contributes to uncontrolled DNA replication.

In the current issue of Acta Haematologica, Jiang et al. [5] analyzed the expression of SAMHD1, together with nine other genes implicated in apoptosis and autophagy in bone-marrow samples of 16 AML and 11 control patients. SAMHD1 expression was not correlated with expression of the nine other genes investigated. While the authors were not able to demonstrate effects of SAMHD1 expression on survival in AML patients, most likely due to the limited number of patients in their study, they reported a significantly lower expression of SAMHD1 in AML as compared to control bone-marrow samples. Hence, this study indicates for the first time that SAMHD1 might suppress leukemogenesis in AML. 
There is preliminary evidence that SAMHD1 affects the disease biology of AML, as evidenced by a significant correlation of SAMHD1 expression and white blood cell count at diagnosis in a cohort of 189 AML patients [2], even though this was not reproduced in the smaller cohort of the present study by Jiang et al. [5]. While current data do not support that cytogenetic or molecular risk factors are correlated with SAMHD1 expression, further studies are warranted to address the following questions: (i) does the lower SAMHD1 expression in AML reflect a likelier leukemogenesis from precursors with endogenously low SAMHD1 levels or is the reduction of SAMHD1 expression an active process during malignant selection? (ii) What are the contributions of the proposed enzyme-dependent (increased dNTP pool) and enzyme-independent (release of stalled DNA replication forks) mechanisms to the development of AML? (iii) Does maintenance of SAMHD1 expression render AML cells particularly vulnerable to therapeutic agents other than ara-C and anthracyclines?

\section{Acknowledgement}

The authors would like to express their gratitude to Jan-Inge Henter and Georgios Rassidakis for scientific support.

\section{Disclosure Statement}

The authors have no conflicts of interest to declare.

\section{Funding Sources}

This work was supported by grants from the Swedish Children's Cancer Foundation (TJ2016-0040, PR2016-0044, PR20180016, and TJ2019-0072 to N.H.); the Swedish Cancer Society (CAN 2017/517 to N.H.;): the Clas Groschinsky Memorial Foundation (M18228 to N.H.); the Mary Béve Foundation for Childhood Cancer Research (to N.H.); the Harald and Greta Jeanssons Foundation (to N.H.); the Åke Wiberg Foundation (M18-0012 to N.H.); the Lars Hierta Memorial Foundation (FO2018-0002 to N.H.); the ìShizu Matsumuraî donation (2018-01086 to N.H.); the Sigurd and Elsa Goljes Memorial Foundation (LA2018-0038 to N.H.); the Stockholm County Council (ALF) (K2892-2016 to N.H); the Felix Mindus contribution to Leukemia Research (201862902715 to N.H.); and the Swedish Society of Medicine (SLS875361 to N.H.). This work was further funded by the German Research Foundation (DFG) (SCHA1950/1-1 to T.S.).

\section{Author Contributions}

The manuscript was conceived by N.H. and written by T.S. and N.H.

\section{References}

1 Herold N, Rudd SG, Ljungblad L, Sanjiv K, Myrberg IH, Paulin CB, et al. Targeting SAMHD1 with the Vpx protein to improve cytarabine therapy for hematological malignancies. Nat Med. 2017 Feb;23(2):256-63.

2 Rassidakis GZ, Herold N, Myrberg IH, Tsesmetzis N, Rudd SG, Henter JI, et al. Low-level expression of SAMHD1 in acute myeloid leukemia (AML) blasts correlates with improved outcome upon consolidation chemotherapy with high-dose cytarabine-based regimens. Blood Cancer J. 2018 Oct;8(11):98.

3 Herold N, Rudd SG, Sanjiv K, Kutzner J, Myrberg $\mathrm{IH}$, Paulin CB, et al. With me or against me: tumor suppressor and drug resistance activities of SAMHD1. Exp Hematol. 2017 Aug; 52:32-9.
4 Coquel F, Silva MJ, Técher H, Zadorozhny K, Sharma S, Nieminuszczy J, et al. SAMHD1 acts at stalled replication forks to prevent interferon induction. Nature. 2018 May; 557(7703):57-61.

5 Jiang H, Li C, Liu Z, Hu R. Expression and Relationship of SAMHD1 with Other Apoptotic and Autophagic Genes in Acute Myeloid Leukemia Patients. Acta Haematol. DOI: $10.1159 / 000500822$. 